Diffusion of Zonal Variables

Using Node-Centered Diffusion Solver

T.-Y. B. Yang

August 15, 2007 
This document was prepared as an account of work sponsored by an agency of the United States Government. Neither the United States Government nor the University of California nor any of their employees, makes any warranty, express or implied, or assumes any legal liability or responsibility for the accuracy, completeness, or usefulness of any information, apparatus, product, or process disclosed, or represents that its use would not infringe privately owned rights. Reference herein to any specific commercial product, process, or service by trade name, trademark, manufacturer, or otherwise, does not necessarily constitute or imply its endorsement, recommendation, or favoring by the United States Government or the University of California. The views and opinions of authors expressed herein do not necessarily state or reflect those of the United States Government or the University of California, and shall not be used for advertising or product endorsement purposes.

This work was performed under the auspices of the U.S. Department of Energy by University of California, Lawrence Livermore National Laboratory under Contract W-7405-Eng-48. 


\title{
Diffusion of Zonal Variables Using Node-Centered Diffusion Solver
}

\author{
Brian Yang \\ University of California, \\ Lawrence Livermore National Laboratory, Livermore CA 94551
}

August 15, 2007

UCRL-TR-233724

\section{Introduction}

Tom Kaiser [1] has done some preliminary work to use the node-centered diffusion solver (originally developed by T. Palmer [2]) in Kull for diffusion of zonal variables such as electron temperature. To avoid numerical diffusion, Tom used a scheme developed by Shestakov et al. [3] and found their scheme could, in the vicinity of steep gradients, decouple nearest-neighbor zonal sub-meshes leading to "alternating-zone" (red-black mode) errors. Tom extended their scheme to couple the sub-meshes with appropriate chosen artificial diffusion and thereby solved the "alternating-zone" problem. Because the choice of the artificial diffusion coefficient could be very delicate, it is desirable to use a scheme that does not require the artificial diffusion but still able to avoid both numerical diffusion and the "alternating-zone" problem. In this document we present such a scheme.

\section{Corner-Centered Variables}

Without loss of generality, let's assume we are solving the diffusion equation for heat conduction:

$$
C_{v} \frac{\partial T}{\partial t}=\nabla \cdot D \nabla T-\sigma T+S_{\text {ext }},
$$

where $T$ is the temperature, $C_{v}$ is the heat capacity per volume, $D$ is the diffusion coefficient, $\sigma$ is the absorption coefficient, and $S_{e x t}$ is the external heat source. In a hydrodynamic code these variables are zone-centered quantities. In order to make use of node-centered diffusion solver, the heat-conduction package keeps its state in the corners, since corners are contained in zones and each corner is uniquely associated with a node that is a vertex of the containing zone. The initial state of heat-conduction package for time step $n+1$ is $T_{C}^{n}$, i.e., the final corner temperature after the heat-conduction package was advanced in time step $n$. Any change in the zonal temperature $T_{Z}$ between the end of heat-conduction at time step $n$ and the beginning of heat-conduction at time step $n+1$ due to other packages such as hydro and ALE is treated as heat source; therefore, the zonal heat source to the heat-conduction package is:

$$
S_{Z}=\frac{C_{v}}{\Delta t}\left[T_{Z}^{\left(n+\frac{1}{2}\right)}-<T_{C}^{n}>_{Z}\right]+S_{e x t, Z}
$$


where $S_{\text {ext,Z }}$ is the zonal heat source that has not been taken into account in the computation of $T_{Z}^{\left(n+\frac{1}{2}\right)}$ and $\langle\ldots\rangle_{Z}$ is the zonal average of a corner-centered variable weighted by the corner volume. The intermediate corner temperature $T_{C}^{\left(n+\frac{1}{2}\right)}$ is then updated as follows:

$$
\begin{aligned}
T_{C}^{\left(n+\frac{1}{2}\right)} & =\left\{\begin{array}{cc}
T_{C}^{n}+S_{Z} \Delta t & , \text { for } S_{Z} \geq 0, \\
\alpha T_{C}^{n} & , \text { for } S_{Z}<0,
\end{array}\right. \\
\alpha & =1+\frac{S_{Z} \Delta t}{<T_{C}^{n}>_{Z}} .
\end{aligned}
$$

It is evident from Eq. (2.3) that $T_{C}^{\left(n+\frac{1}{2}\right)}$ satisfies the following energy conservation relation:

$$
C_{v}<T_{C}^{\left(n+\frac{1}{2}\right)}>_{Z}=C_{v} T_{Z}^{\left(n+\frac{1}{2}\right)}+S_{e x t, Z} \Delta t
$$

\section{Solving Corner Variables Using Node-Centered Diffusion Solvers}

The diffusion-absorption equation for each corner with implicit finite-difference in time is

$$
C_{v, i} T_{C, i}^{(n+1)}-\Delta t\left[\nabla_{c} \cdot D \nabla_{c} T_{C, i}^{(n+1)}\right]=C_{v, i} T_{C, i}^{\left(n+\frac{1}{2}\right)}-\Delta t \sigma_{i} T_{C, i}^{(n+1)},
$$

where, $C_{v, i}$ is the heat capacity per volume of the zone containing the corner. Although, the differential operator $\nabla_{c}$ for the corner is not explicitly defined, we postulate that the nodal averaged operator

$$
\nabla \cdot D \nabla T_{N}^{(n+1)} \equiv \frac{\sum_{i=c_{1}, c_{2}, \cdots} V_{i} \nabla_{c} \cdot D \nabla_{c} T_{C, i}^{(n+1)}}{\sum_{i=c_{1}, c_{2}, \cdots} V_{i}}
$$

can be approximated by a nodal discretization. In order to use node-centered diffusion solver, we have introduced nodal temperatures:

$$
T_{N}^{k} \equiv \frac{\sum_{i=c_{1}, c_{2}, \cdots} C_{v, i} V_{i} T_{C, i}^{k}}{\sum_{i=c_{1}, c_{2}, \cdots} C_{v, i} V_{i}}, \quad k=\left(n+\frac{1}{2}\right) \text { or }(\mathrm{n}+1) .
$$

Here, $\sum_{i=c_{1}, c_{2}, \cdots}$ stands for summation over all the corners surrounding the node and $V_{i}$ is the volume of a given corner. Volume-weighted average of Eq. (3.6) gives the nodal equation

$$
\begin{aligned}
<C_{v}>_{N} T_{N}^{(n+1)}-\Delta t\left[\nabla \cdot D \nabla T_{N}^{(n+1)}\right] & =<C_{v}>_{N} T_{N}^{\left(n+\frac{1}{2}\right)}-\Delta t \bar{\sigma}_{N} T_{N}^{(n+1)}, \\
<C_{v}>_{N} & =\frac{\sum_{i=c_{1}, c_{2}, \cdots} C_{v, i} V_{i}}{\sum_{i=c_{1}, c_{2}, \cdots} V_{i}} . \\
\bar{\sigma}_{N} \equiv & \frac{<C_{v}>_{N} \sum_{i=c_{1}, c_{2}, \cdots} V_{i} \sigma_{i} T_{C, i}^{(n+1)}}{\sum_{i=c_{1}, c_{2}, \cdots} V_{i} C_{v, i} T_{C, i}^{(n+1)}} .
\end{aligned}
$$


Notice that, the calculation of $\bar{\sigma}_{N}$ requires knowledge of the relative amplitudes of corner variables $T_{C, i}^{(n+1)}$ for a given node. Since the absorption coefficient is most important in the non-diffusive limit $(D=0)$, we assume that $T_{C, i}^{(n+1)}$ for the corners surrounding a particular node are proportional to the non-diffusive solution $\left(T_{C, i}^{N D}\right)$ of Eq. (3.6):

$$
\begin{aligned}
T_{C, i}^{N D} & =\frac{T_{C, i}^{\left(n+\frac{1}{2}\right)}}{1+\eta_{i}} \\
\eta_{i} & =\frac{\sigma_{i} \Delta t}{C_{v, i}} .
\end{aligned}
$$

The nodal absorption coefficient $\bar{\sigma}_{N}$ can then be approximated by

$$
\begin{aligned}
\bar{\sigma}_{N} \approx & \frac{\left\langle C_{v}>_{N} \sum_{i=c_{1}, c_{2}, \cdots} \frac{V_{i} \sigma_{i} T_{C, i}^{\left(n+\frac{1}{2}\right)}}{1+\eta_{i}}\right.}{\sum_{i=c_{1}, c_{2}, \cdots} \frac{V_{i} C_{v, i} T_{C, i}^{\left(n+\frac{1}{2}\right)}}{1+\eta_{i}}}=\frac{\sum_{i=c_{1}, c_{2}, \cdots} \frac{V_{i} \sigma_{i} T_{C, i}^{\left(n+\frac{1}{2}\right)}}{1+\eta_{i}}}{\hat{T}_{N} \sum_{i=c_{1}, c_{2}, \cdots} V_{i}} \\
\hat{T}_{N} & =\frac{\sum_{i=c_{1}, c_{2}, \cdots} V_{i} C_{v, i} \frac{T_{C, i}^{\left(n+\frac{1}{2}\right)}}{1+\eta_{i}}}{\sum_{i=c_{1}, c_{2}, \cdots} V_{i} C_{v, i}} .
\end{aligned}
$$

After solving the nodal equation (3.9) for the new nodal temperature $T_{N}^{(n+1)}$, the corner temperatures are then updated as follows

$$
T_{C, i}^{\prime(n+1)}=\left\{\begin{array}{cl}
\frac{T_{C, i}^{\left(n+\frac{1}{2}\right)}}{1+\eta_{i}}+T_{N}^{(n+1)}-\hat{T}_{N} & , \text { for } T_{N}^{(n+1)} \geq \hat{T}_{N}, \\
\frac{T_{N}^{(n+1)}}{\hat{T}_{N}} \frac{T_{C, i}^{\left(n+\frac{1}{2}\right)}}{1+\eta_{i}} & , \text { for } T_{N}^{(n+1)}<\hat{T}_{N} .
\end{array}\right.
$$

It is evident that $T_{C, i}^{\prime}$ defined in Eq. (3.16) satisfies the following equation

$$
\sum_{i=c_{1}, c_{2}, \cdots} V_{i} C_{v, i} T_{C, i}^{\prime(n+1)}=T_{N}^{(n+1)} \sum_{i=c_{1}, c_{2}, \cdots} V_{i} C_{v, i} .
$$

Furthermore, in the non-diffusive limit $(D=0)$, solution of Eq. (3.9) is

$$
T_{N}^{(n+1)}=\frac{<C_{v}>_{N} T_{N}^{\left(n+\frac{1}{2}\right)}}{<C_{v}>_{N}+\Delta t \bar{\sigma}_{N}},
$$

which can be shown from Eqs. (3.8) and (3.14) equal to $\hat{T}_{n}$ defined in Eq. (3.15). Hence, $T_{C, i}^{\prime(n+1)}$ reduces to the non-diffusive limit in Eq. (3.12).

If the heat capacity-weighted average of $T_{C}^{\prime(n+1)}$ were to be used as the final zonal temperature of time step $n+1$, when the diffusion coefficient is non-zero, the present scheme would suffer the same "alternating-zone" errors as the scheme by Shestakov et al. To eliminate the "alternatingzone" errors, $T_{C}^{\prime(n+1)}$ is relaxed through a sub-nodal temperature equilibration process as described in Sec. (4). 


\section{Sub-Nodal Temperature Equilibration}

We postulate that within a nodal control volume, the corner temperature of each corner satisfies the following equation

$$
\begin{aligned}
C_{v, i} \frac{d T_{C, i}}{d t} & =-\mu_{i}\left(T_{C, i}-\tilde{T}\right)-\sigma_{i}\left[T_{C, i}-T_{C, i}^{\prime(n+1)}\right]+Q_{C, i} \\
\mu_{i} & =D_{i} / L_{i}^{2}
\end{aligned}
$$

where $L_{i}$ is the scale length of corner $i$ and $Q_{C, i}=C_{v, i}\left[T_{C, i}^{\prime(n+1)}-T_{C, i}^{n}\right] / \Delta t$ is the heat source taking into account all the processes, including diffusion, that cause the change in the corner temperature so far, from $T_{C, i}^{n}$ to $T_{C, i}^{(n+1)}$. For energy conservation, the reference temperature $\tilde{T}$ must satisfy

$$
\tilde{T} \sum_{i=c_{1}, c_{2}, \cdots} V_{i} \mu_{i}=\sum_{i=c_{1}, c_{2}, \cdots} V_{i}\left[\left(\mu_{i}+\sigma_{i}\right) T_{C, i}-\sigma_{i} T_{C, i}^{\prime(n+1)}\right]
$$

With implicit finite-difference in time, Eqs. (4.19) and (4.21) reduce to

$$
\begin{aligned}
\left(C_{v, i}+\sigma_{i} \Delta t+\mu_{i} \Delta t\right) T_{C, i}^{(n+1)} & =\left(C_{v, i}+\sigma_{i} \Delta t\right) T_{C, i}^{\prime(n+1)}+\mu_{i} \Delta t \tilde{T} \\
\tilde{T} & =\frac{\sum_{i=c_{1}, c_{2}, \cdots} V_{i}\left[\left(\mu_{i}+\sigma_{i}\right) T_{C, i}^{(n+1)}-\sigma_{i} T_{C, i}^{\prime(n+1)}\right]}{\sum_{i=c_{1}, c_{2}, \cdots} V_{i} \mu_{i}} .
\end{aligned}
$$

Substituting Eq. (4.22) into Eq. (4.23), we eliminate the unknowns $T_{C, i}^{(n+1)}$ and obtain the expression for $\tilde{T}$

$$
\tilde{T}=\frac{\sum_{i=c_{1}, c_{2}, \cdots} V_{i} \frac{C_{v, i} \mu_{i} \Delta t T_{C, i}^{\prime(n+1)}}{C_{v, i}+\left(\sigma_{i}+\mu_{i}\right) \Delta t}}{\sum_{i=c_{1}, c_{2}, \cdots} V_{i} \frac{C_{v, i} \mu_{i} \Delta t}{C_{v, i}+\left(\sigma_{i}+\mu_{i}\right) \Delta t}} .
$$

Eqs. (4.22) and (4.24) together give the final corner temperature $T_{C, i}^{(n+1)}$. The final zonal temperature of time step $n+1$ is the heat capacity-weighted average of $T_{C, i}^{(n+1)}$.

\section{$5 \quad$ Numerical Results}

A one-dimensional heat-conduction test problem was set up to test the algorithm. The mesh was a 1 - $\mathrm{cm}$ cube with 1 zone in the $x$ and $y$ directions and 20 equally sized zones in the $z$ direction. The initial temperature profile was a spike with the temperature of $100 \mathrm{keV}$ for the zone with the smallest $z$-coordinate and 0 for the rest of the zones. Reflecting boundary condition was applied at all the boundaries. The heat capacity per volume was $C_{v}=5.0 \mathrm{jerks} \cdot \mathrm{keV}^{-1} \mathrm{~cm}^{-3}$, the diffusion coefficient was $D=1.25 \mathrm{jerks} \cdot \mathrm{keV}^{-1} \mathrm{~cm}^{-1} \mathrm{sh}^{-1}$, and there was no absorption. Shown in Fig. 1 are the temperature profiles at the end of 20 cycles with $5 \times 10^{-3} \mathrm{sh}$ time step $(t=0.1 \mathrm{sh})$ calculated using 4 different methods: 
1. Curve A (red dotted curve): Analytical result

$$
T(z, t)=100 \mathrm{keV} \frac{2 \Delta z}{\sqrt{4 \pi D t / C_{v}}} \exp \left(-\frac{z^{2} C_{v}}{4 D t}\right),
$$

where $\Delta z=0.05 \mathrm{~cm}$ is the zone width in the $z$-direction.

2. Curve B (blue solid curve): Current algorithm with sub-nodal equilibration.

3. Curve C (green dashed curve): Current algorithm without sub-nodal equilibration.

4. Curve D (black dotted-dashed curve): In every cycle, interpolate the zonal temperatures to the nodes, solving the nodal temperatures with the nodal diffusion solver, and interpolate the nodal temperatures back to the zones.

Comparing curves A and B in Fig. 1, it is evident that the result from the current algorithm with the sub-nodal equilibration is in excellent agreement with the analytical result. The "alternatingzone" error can be clearly seen in curve C, at the two leftmost zones, where there was a steep gradient in the initial temperature profile. The usage of the corner variables in the current algorithm, even without the sub-nodal equilibration, produces a smooth temperature profile in zones where the gradient of the initial temperature is 0. In contrast, Tom Kaiser [1] showed that using the scheme by Shestakov et al. the "alternating-zone" error spread out to zones that had zero gradient in initial temperature. Finally, Curve D shows that interpolation between the zonal temperatures and the nodal temperature every cycle is too diffusive.

Another test problem examined the capability of the current algorithm to preserve the spherical symmetry of the impulse response on Kershaw's "z-mesh" in 2D-RZ geometry. The geometry of the test problem was a 1-cm square in the $r-z$ coordinate system (see Fig. 2). The lower boundary of the problem was on the $z$-axis. Reflecting boundary condition was applied at all the boundaries. The heat capacity per volume was $C_{v}=5.0 \mathrm{jerks} \cdot \mathrm{keV}^{-1} \mathrm{~cm}^{-3}$, the diffusion coefficient was $D=0.75 \mathrm{jerks} \cdot \mathrm{keV}^{-1} \mathrm{~cm}^{-1} \mathrm{sh}^{-1}$, and there was no absorption. The initial temperature profile was a spike with the temperature of $100 \mathrm{keV}$ for the zone located at the the lower-left corner and 0 for the rest of the zones. The time step for each cycle is $2 \times 10^{-3} \mathrm{sh}$. Shown in Figs. 2 and 3 are the contour plots of the temperatures at cycle $100(t=0.2 s h)$ and cycle $200(t=0.4 s h)$, respectively, using VisIt (http://www.llnl.gov/visit/). To better quantify the degree of deviation from the spherical symmetry, the "lineout" mode in VisIt was used to trace the temperature as a function of distance from origin (lower-left corner). Shown in Figs. 4 and 5 are the "lineout" plots at cycle 100 and cycle 200 , respectively, for three angles, $0^{\circ}$ ( $z$-axis), $90^{\circ}$ ( $r$-axis), and $45^{\circ}$. The curves in Figs. 4 and 5 are:

1. Curve A (black solid curve): $0^{\circ}$.

2. Curve B (red solid curve): $90^{\circ}$.

3. Curve C (blue solid curve): $45^{\circ}$.

4. Curve D (green dotted curve): analytical solution

$$
T(d, t)=100 \mathrm{keV} \frac{2 V_{0}}{\left(4 \pi D t / C_{v}\right)^{3 / 2}} \exp \left(-\frac{d^{2} C_{v}}{4 D t}\right),
$$


where $d$ is the distance from the origin and $V_{0}=\pi \times 10^{-6} \mathrm{~cm}^{3}$ is the volume of the zone at the lower-left corner.

The jaggedness of the "lineout" curves is expected, because both Kull and VisIt treat the temperature as a spatially uniform value within each zone. The importance point is that the "lineout" curves do not deviate much from the analytical solution. The $45^{\circ}$ curves on both Figs. 4 and 5 do not monotonically decrease. The inversion of the slope is caused by the interaction between the "lineout" operator and the severe skewness of the "z-mesh". As the "lineout" operator traverses from the origin along the $45^{\circ}$-line, it may intercept a zone centered at a larger distance from the origin before it intercepts another zone centered at a smaller distance, resulting in an inversion of the slope. For a similar reason, the contours in Figs. 2 and 3 also show some jaggedness but in a much lesser level.

\section{References}

[1] T.B. Kaiser, "Evolution of a Zone-Centered Field with a Node-Centered Diffusion Solver on an Unstructured Mesh," American Physical Society, 44th Annual Meeting of the Division of Plasma, abstract \#BP1.109 (2002).

[2] T. Palmer, "Discretization the Diffusion Equation on Unstructured Polygonal Meshes in Two Dimensions," Annals of Nuclear Energy 28, 1851 (2001).

[3] A.I. Shestakov, J.L. Milovich, and M.K. Prasad, "Combining Cell and Point Centered Methods in 3D Unstructured-Grid Radiation-Hydrodynamic Codes," J. Comput. Phys. 170, 81 (2001). 


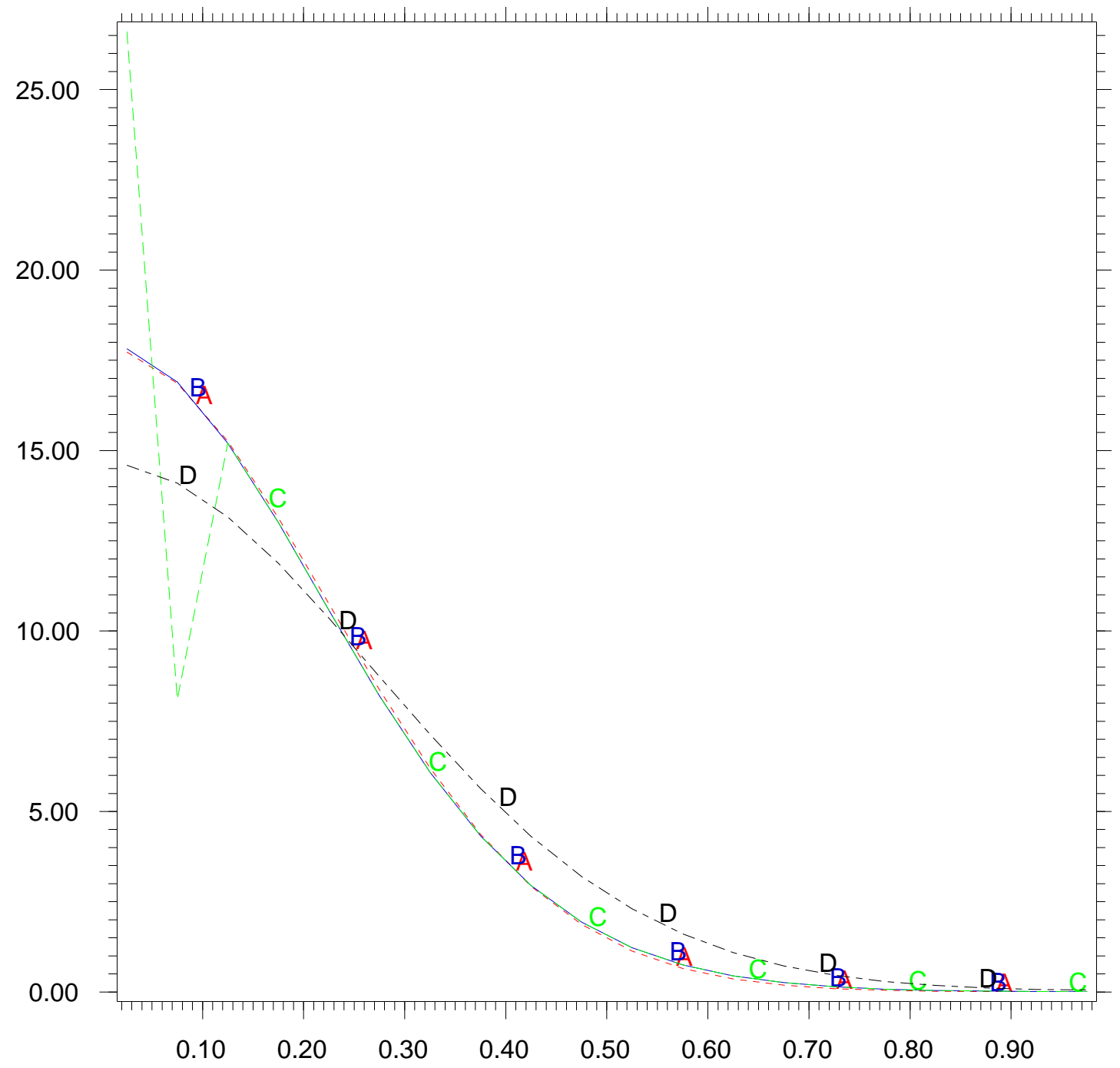

Figure 1: Temperature (in $\mathrm{keV}$ ) vs. $z$ (in $\mathrm{cm}$ ) at the end of 20 cycles $\left(t=2.0 \times 10^{-2} \mathrm{sh}\right.$ ) calculated with 4 different methods. 


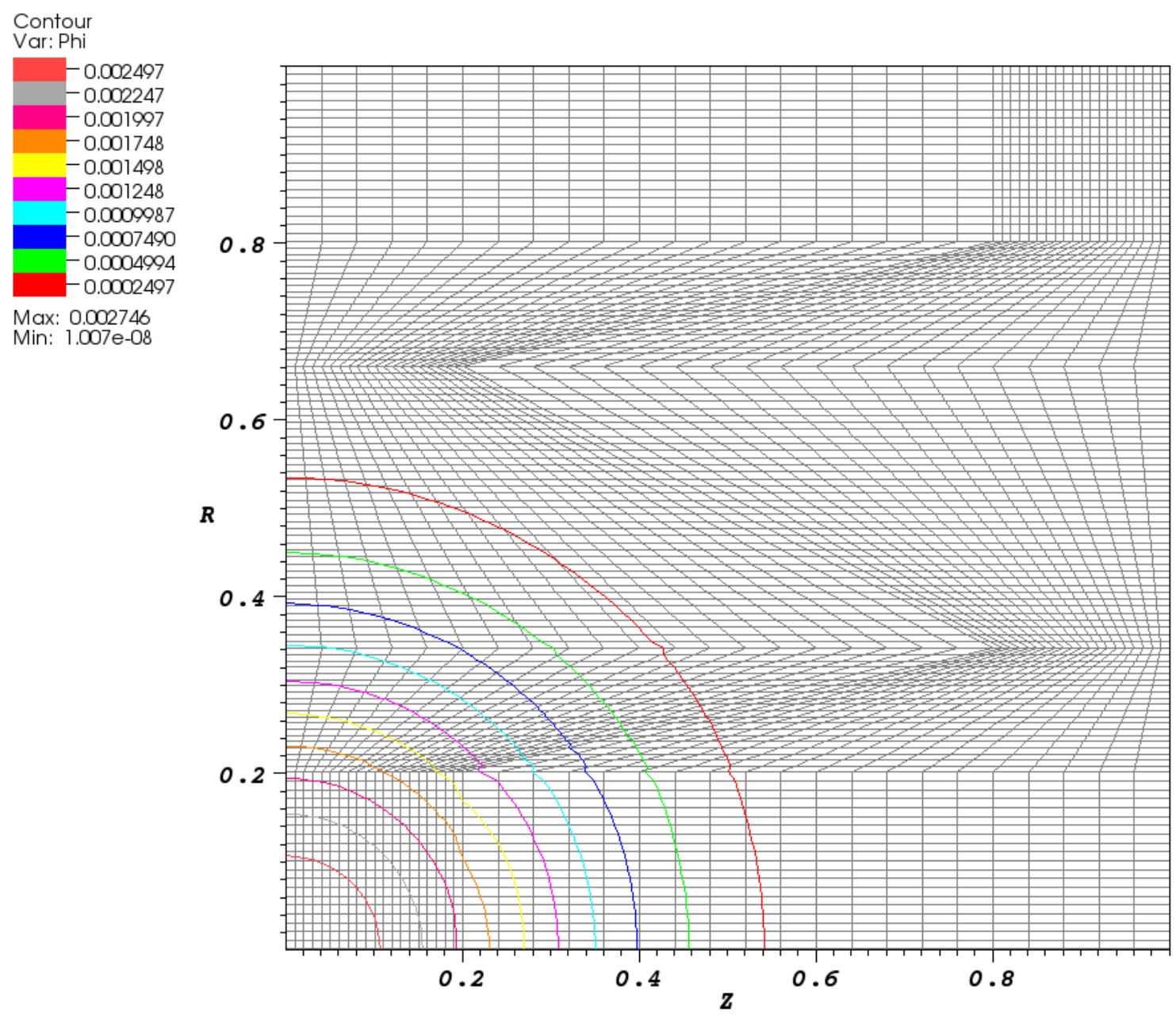

Figure 2: Contour plot of the temperature (in $\mathrm{keV}$ ) at cycle 100 for the "z-mesh" test problem. 


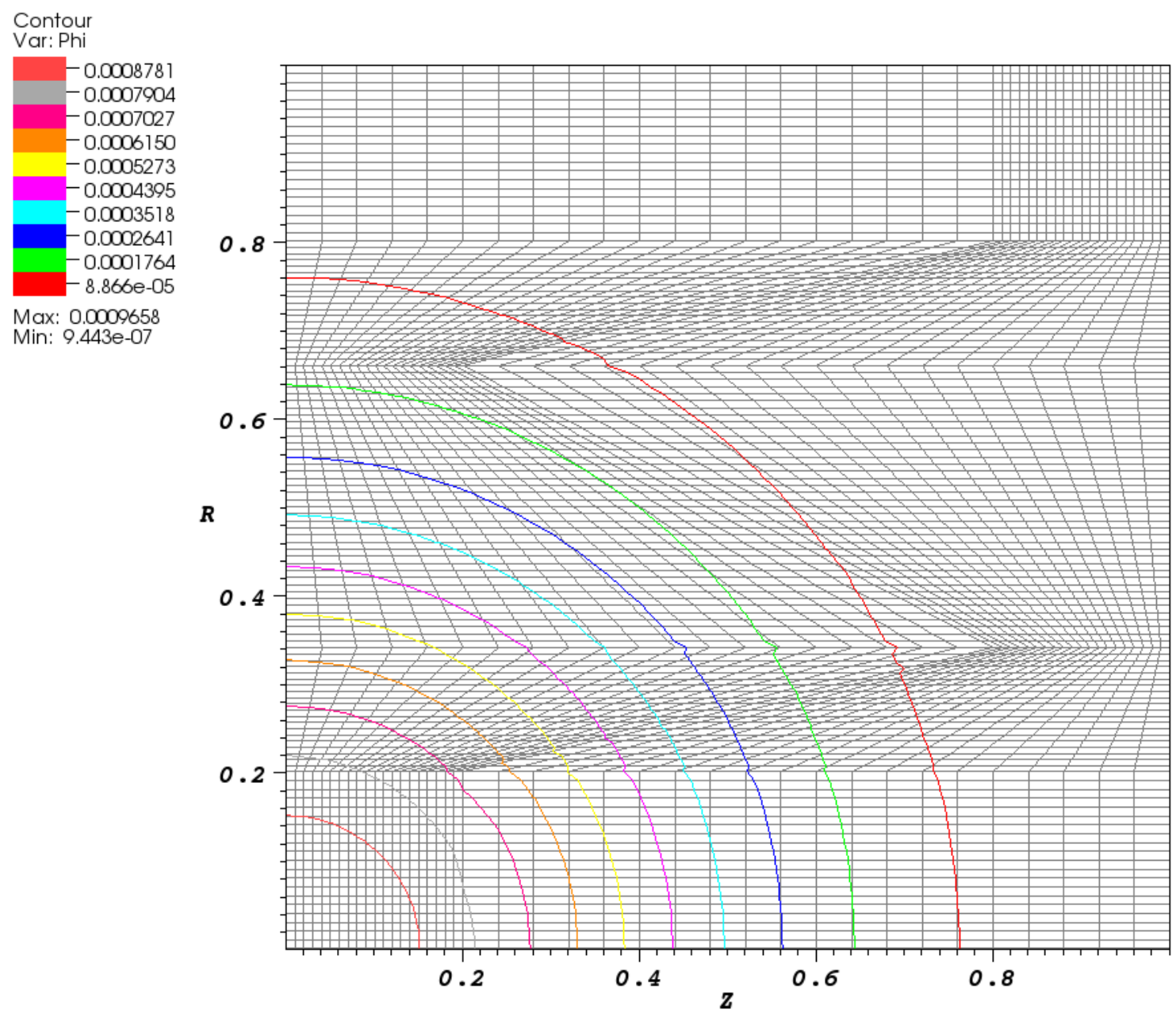

Figure 3: Contour plot of the temperature (in $\mathrm{keV}$ ) at cycle 200 for the "z-mesh" test problem. 


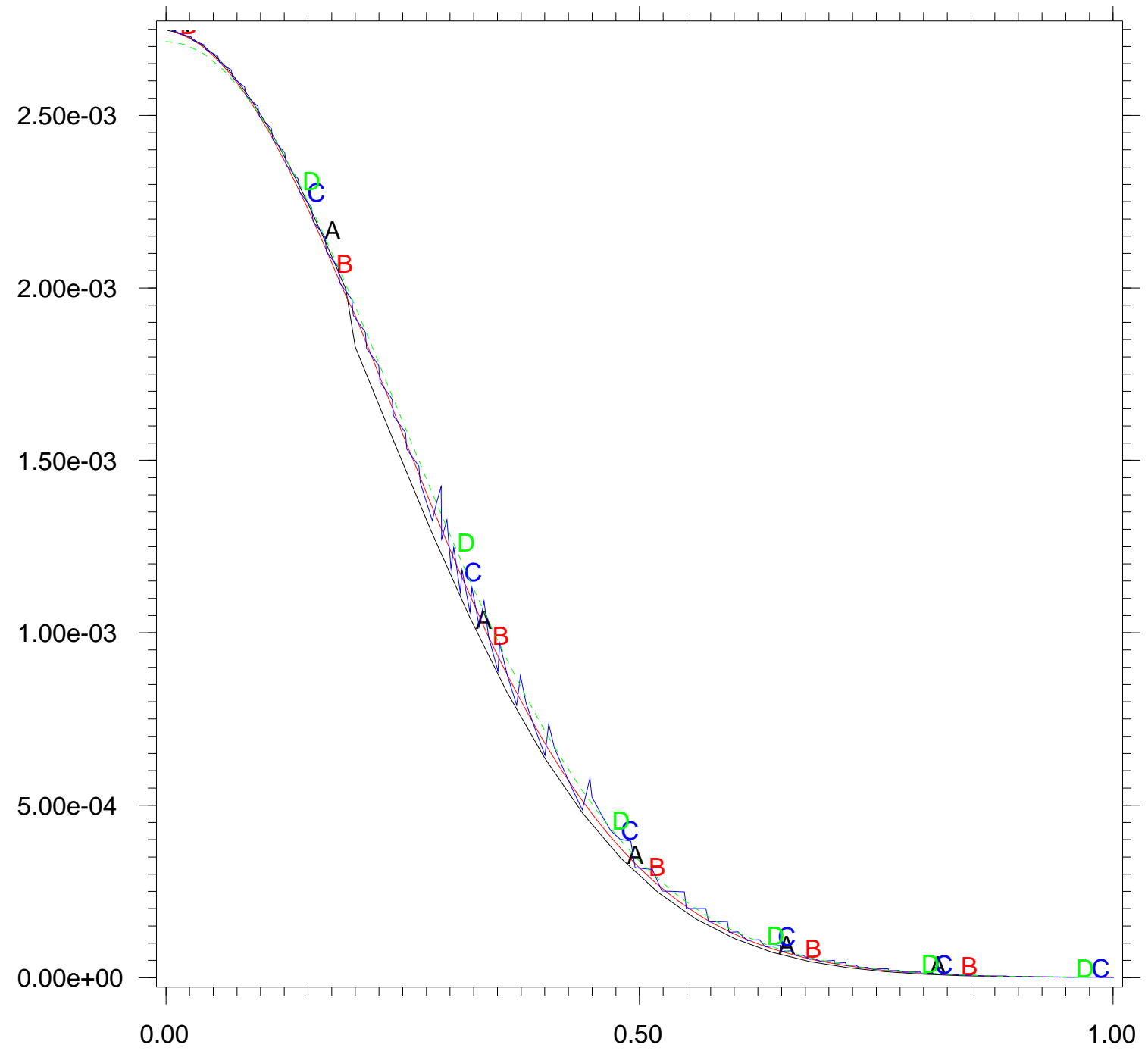

Figure 4: "Lineout" curves and the analytical solution, temperature (in $k e V$ ) vs. $d$ (in $\mathrm{cm}$ ), at cycle 100 for the "z-mesh" test problem. 




Figure 5: "Lineout" curves and the analytical solution, temperature (in $k e V$ ) vs. $d$ (in $\mathrm{cm}$ ), at cycle 200 for the "z-mesh" test problem. 